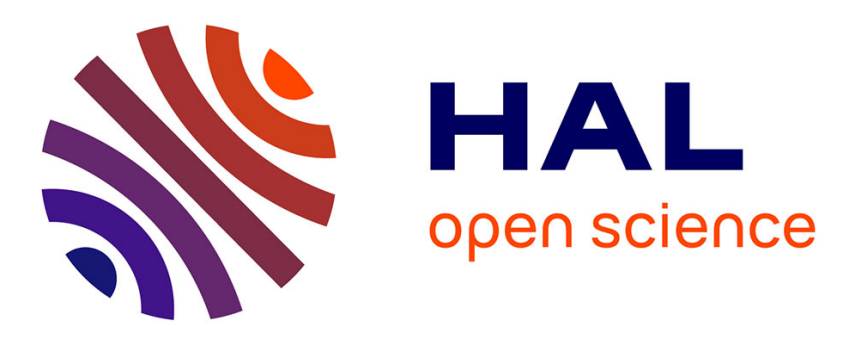

\title{
Dispersion truncation affects the phase behavior of bulk and confined fluids: Coexistence, adsorption, and criticality
}

\author{
Alexander Schlaich, Benoit Coasne
}

\section{- To cite this version:}

Alexander Schlaich, Benoit Coasne. Dispersion truncation affects the phase behavior of bulk and confined fluids: Coexistence, adsorption, and criticality. Journal of Chemical Physics, 2019, 150 (15), pp.154104. 10.1063/1.5085431 . hal-02328646

\section{HAL Id: hal-02328646 \\ https://hal.science/hal-02328646}

Submitted on 5 Nov 2020

HAL is a multi-disciplinary open access archive for the deposit and dissemination of scientific research documents, whether they are published or not. The documents may come from teaching and research institutions in France or abroad, or from public or private research centers.
L'archive ouverte pluridisciplinaire HAL, est destinée au dépôt et à la diffusion de documents scientifiques de niveau recherche, publiés ou non, émanant des établissements d'enseignement et de recherche français ou étrangers, des laboratoires publics ou privés. 
Dispersion truncation affects the phase behavior of bulk and confined fluids: coexistence, adsorption and criticality

Alexander Schlaich ${ }^{1, \text { a) }}$ and Benoit Coasne ${ }^{1, b)}$

Univ. Grenoble Alpes, CNRS, LIPhy, 38000 Grenoble, France

(Dated: December 6, 2018)

We present molecular simulations of bulk and confined Lennard-Jones fluids to asses the effect of dispersion truncation through a simple spherical cutoff. The latter is well corrected on a mean field level for bulk fluids if the cutoff distance is larger than about three molecular diameters. In confinement, however, there is no general analytical treatment and thus the truncated and shifted Lennard-Jones potential has to be employed, with drastic consequences on the bulk critical temperature, vapor/liquid coexistence pressure and surface tension. We show using Grand-Canonical Monte-Carlo simulations of nitrogen adsorption in amorphous silica nanopores that the choice of the cutoff significantly modifies the pressure at which capillary condensation occurs and compute the capillary critical temperature in terms of a first order transition between an adsorbed film and filled pores.

\footnotetext{
a)Electronic mail: alexander.schlaich@univ-grenoble-alpes.fr

b)Electronic mail: benoit.coasne@univ-grenoble-alpes.fr
} 


\section{INTRODUCTION}

Nanoporous materials are widely used in industry as adsorbents for filtration, separation and catalysis, where understanding the thermodynamics and transport of the confined fluids is crucial. Typically, fluids confined within nanometric pores (the size of a few molecular diameters) exhibit properties that are significantly different from their bulk counterpart. In particular, surface forces and the reduced dimensions of the system affect phase transitions due to capillary condensation, freezing, etc. For obtaining microscopic insights on the confinement effects, computer simulations have proven as helpful tool and shall be discussed in the present article in the context of predicting the thermodynamics of the confined systems.

For a typical pair potential, the computational cost increases roughly as $N^{2}$ with $N$ being the number of particles in a simulation. ${ }^{1}$ A tremendous reduction can be achieved by assuming that the largest contribution to the potential and forces is due to the closest neighbors only. Then, a spherical cutoff is typically applied. This means, that the pair potential $\phi(r)$ is set to zero for $r \geq r_{\text {cut }}$, where $r_{\text {cut }}$ is the cutoff distance. In this article, we focus on the Lennard-Jones (LJ) potential, which is probably the most widely studied intermolecular interaction potential in computer simulations and performs realistically for small spherical and nonpolar molecules. It has been employed since more than 50 years in order to study phase equilibria as well as interfacial and transport properties. ${ }^{2-5}$ The $\mathrm{LJ}$ potential is given by

$$
\phi(r)=4 \varepsilon\left[\left(\frac{\sigma}{r}\right)^{12}-\left(\frac{\sigma}{r}\right)^{6}\right]
$$

where $r$ is the distance between two interacting particles, $\varepsilon$ is the interaction strength and $\sigma$ determines the equilibrium separation. Equation (1) typically is truncated at a value $r_{\text {cut }} \leq L / 2$, where $L$ is the (shortest) length of the simulation box. In a bulk liquid, and for sufficiently large values of $r_{\text {cut }}$, the pair correlation function can be assumed to be unity, $g(r)=1$ for $r>r_{\text {cut }}$. Integrating Eq. (1) from $r_{\text {cut }}$ to $\infty$ leads to the so-called long-range tail corrections (LRC) for the energy $U$ and pressure $P .{ }^{1}$ Throughout this paper. we use reduced units in which the density is measured in terms of the particle diameter $\sigma$ as $\tilde{\rho}=(N / V) \sigma^{3}$, with $N$ the number of particles in the volume $V$. All energies are measured in terms of thermal energy $k_{\mathrm{B}} T$ and, correspondingly, the reduced temperature and pressure follow as $\tilde{T}=k_{\mathrm{B}} T / \varepsilon$ and $\tilde{P}=P \varepsilon / \sigma^{3}$. The LRC for the potential energy 
and pressure then read as

$$
\begin{aligned}
& \tilde{U}_{\text {lrc }}=\frac{8}{9} \pi \tilde{\rho}^{2}\left[\frac{1}{\tilde{r}_{\text {cut }}^{9}}-\frac{3}{\tilde{r}_{\text {cut }}^{3}}\right], \\
& \tilde{P}_{\text {lrc }}=\frac{32}{9} \pi \tilde{\rho}^{2}\left[\frac{1}{\tilde{r}_{\text {cut }}^{9}}-\frac{3}{2 \tilde{r}_{\text {cut }}^{3}}\right] .
\end{aligned}
$$

Extensions of Eqs. (2) and (3) to non-isotropic systems can be made only for simplifying geometries, like a planar interface. ${ }^{6,7}$ Thus, in practice, when anisotropic heterogeneous systems are simulated, the truncated and shifted LJ potential is employed,

$$
\phi_{\mathrm{ts}}(r)= \begin{cases}\phi(r)-\phi\left(r_{\mathrm{cut}}\right) & \text { if } \quad r \leq r_{\mathrm{cut}} \\ 0 & \text { if } \quad r>r_{\mathrm{cut}} .\end{cases}
$$

The shift $-\phi\left(r_{\text {cut }}\right)$ is necessary to ensure consistent thermodynamic behavior ${ }^{8}$ and a constant chemical potential of the particles in the simulations. ${ }^{9,10}$

In this work, using Monte Carlo (MC) and molecular dynamics (MD) simulations, we study in Section II the consequences of using Eq. (4) instead of Eq. (1) for bulk thermodynamic properties such as the critical temperature, liquid/vapor saturation pressure and surface tension, with particular impact on the choice of $r_{\text {cut }}$ and confirm that the mean field relations given in Eqs. (2) and (3) yield accurate predictions for the shift in the bulk critical temperature $T_{\mathrm{c}}$, the vapor/liquid saturation pressure $P_{\text {sat }}$ and the surface tension $\gamma$ due to the cutoff. We then turn to the impact of $r_{\text {cut }}$ on adsorption isotherms. Among porous solids with pores at the nanometer scale, silicabased materials are one of the most common and nitrogen adsorption experiments at low temperature are routinely used for the characterization of porous solids. ${ }^{11-17}$ We perform corresponding Grand Canonical Monte Carlo (GCMC) simulations in Section III. In the latter, particles are exchanged with a virtual reservoir of ideal gas particles at pressure $P=f / \varphi$, with the fugacity $f=1 /\left(\beta \Lambda^{3}\right) \exp (\beta \mu)$ that relates the chemical potential $\mu$ to $P$ via the fugacity coefficient $\varphi$, $\beta=1 / k_{\mathrm{B}} T$ is the reduced temperature and $\Lambda$ the de Broglie wavelength. Obviously, $\varphi=1$ for the ideal gas, but in general $\varphi=\varphi(P, T)$, which we derive based on the LJ equation of state (EOS) in Section II D. Finally, in Section IV we discuss the temperature $T_{\mathrm{cc}}$ below which capillary condensation is observed in dependency of pore width and cutoff. We show that for a given $r_{\text {cut }}, T_{\text {cc }}\left(r_{\text {cut }}\right)$ is related to $T_{\mathrm{c}}\left(r_{\text {cut }}\right)$, allowing to extrapolate to $r_{\text {cut }} \rightarrow \infty$ in which case the full LJ potential Eq. (1) is recovered. In Section V we summarize our results and draw conclusions which are also relevant to the experimental analysis of adsorption isotherms. 


\section{BULK FLUID PROPERTIES}

\section{A. Coexistence line \& critical temperature}

We perform Gibbs ensemble Monte Carlo (GEMC) simulations ${ }^{18,19}$ using MCCCS Towhee. $^{20}$ In summary, GEMC simulations mimic two coexisting simulation boxes of volume $V^{\mathrm{I}}+V^{\mathrm{II}}=V$ consisting of $N^{\mathrm{I}}+N^{\mathrm{II}}=N$ particles, respectively. While $V^{\mathrm{I}}, V^{\mathrm{II}}, N^{\mathrm{I}}$ and $N^{\mathrm{II}}$ fluctuate, $N$ and $V$ are constant in the (NVT) ensemble employed. Three distinct types of trial moves are allowed for a Monte Carlo step: particle displacement, volume rearrangement, and particle exchange. ${ }^{21}$ Our GEMC simulations are performed for $N=1000$ up to $1500 \mathrm{LJ}$ particles, where $V$ was adjusted such that about $20 \%$ of the particles are in the vapor phase.

Figure 1 (A) shows the coexistence densities obtained from GEMC simulations for cutoff radii $r_{\text {cut }}=3 \sigma, 4 \sigma$ and $5 \sigma$ (blue circles, red squares and green triangles, respectively). The coexistence curves depend sensitively on $r_{\text {cut }}$ if the truncated and shifted potential given in Eq. (4) is used, as has been noted at several places before in the past and current literature (see e.g. Refs. ${ }^{9,22-27}$ ). The hollow black symbols included in Fig. 1 (A) show the corresponding results of the GEMC simulations if the LJ potential Eq. (1) together with the tail corrections Eqs. (2) and (3) is employed. The corresponding data points in Fig. 1 (A) including the LRC converge excellently for all studied values of $r_{\text {cut }}$. We employ the Modified Benedict-Webb-Rubin equation of state (MBWR) by Johnson et al. ${ }^{22,28}$ for the equation of state (EOS), $P(\rho)$. Although a multitude of models has emerged over time for the LJ EOS, see e.g. Ref. ${ }^{29}$ for a current overview, we decided to employ the MBWR model from Johnson et al. because of its wide use in literature. Deviations for the saturating vapor pressures $P_{\text {sat }}$ and vapor density $\rho(P)$, which are the important quantities for our further analysis, are small between different models.

The critical temperature and density are given by the saddle point,

$$
\begin{aligned}
&\left.\left(\frac{\partial P}{\partial \rho}\right)_{T}\right|_{T_{\mathrm{c}}, \rho_{\mathrm{c}}}=0 \\
& \text { and }\left.\quad\left(\frac{\partial^{2} P}{\partial \rho^{2}}\right)_{T}\right|_{T_{\mathrm{c}}, \rho_{\mathrm{c}}}=0,
\end{aligned}
$$

which we obtain numerically from the EOS, c.f. the solid black line in Fig. 1 (A), in perfect agreement with our GEMC simulation data. For the truncated-shifted potential, we include the tail correction given by Eq. (3) to the numerical solution of Eqs. (5) and (6), resulting in the solid colored lines shown in Fig. 1 (A), which agree remarkably with the simulation data for $r_{\text {cut }}=4 \sigma$ 

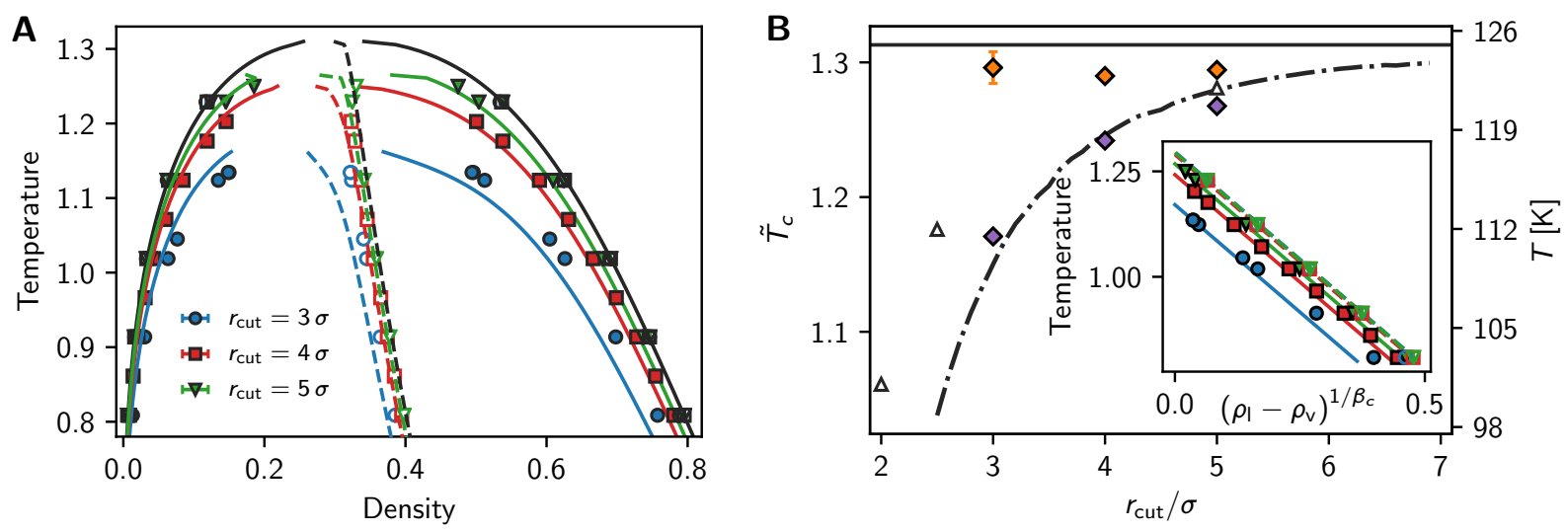

Figure 1. Liquid/vapor coexistence curve and critical temperature. (A) Coexistence data for $r_{\text {cut }}=3 \sigma$ (circles), $4 \sigma$ (squares) and $5 \sigma$ (triangles) for the truncated and shifted potential given in Eq. (4) (filled symbols). Hollow symbols denote the corresponding simulation data if Eq. (1) with the LRC is employed. Solid lines show the MWBR equation of state from Johnson et al. ${ }^{22}$ (black line) and the mean field corrections for finite cutoff (colored lines). Colored hollow symbols and dashed lines show the rectilinear density as given by the 1.h.s. of Eq. (8). (B) Critical temperature for the LJ fluid determined from our simulations using the truncated and shifted potential (purple diamonds) and using the LRC (orange diamonds). Empty triangles denote simulation results from Panagiotopoulos. ${ }^{23}$ Horizontal black line shows the value $\tilde{T}_{\mathrm{c}}=1.313$ of the MWBR EOS, dashed orange line $T^{\star}=1.298$ resulting from the hyperscaling relation of the surface tension discussed below. Dash dotted line represents the MWBR mean field prediction. Right axis shows the temperature for the LJ parameters of nitrogen for comparison. Inset: Fits of Eq. (7) to the simulation data using the Ising critical exponent $\beta_{\mathrm{c}}=0.32$ for the cut and shifted potential (solid lines, filled symbols) and the LRC (dashed lines, hollow symbols). Color encoding is the same as in (A).

and $5 \sigma$. However, for $r_{\text {cut }}=3 \sigma$, the data slightly deviate due to the breakdown of the mean field assumption $g(r)=1$ for $r>r_{\text {cut }}$ when such a small cutoff is used.

From the simulation data shown in Fig. 1 (A) we determine the critical temperature $\tilde{T}_{\mathrm{c}}$ from the scaling law

$$
\rho_{\mathrm{sat}}^{1}-\rho_{\mathrm{sat}}^{\mathrm{v}}=B\left|T-T_{\mathrm{c}}\right|_{c}^{\beta}
$$

where $\rho_{\text {sat }}^{1}, \rho_{\text {sat }}^{\mathrm{v}}$ are the liquid and vapor densities at coexistence, $\beta_{\mathrm{c}}=0.32$ is the Ising critical exponent and $B$ is a fitting parameter. Fits of Eq. (7) to the simulation data are shown in the inset of Fig. 1 (B) for the truncated and shifted potential (filled symbols and solid lines) and using the LRC (empty symbols and dashed lines). As has been noted extensively in the literature, 
finite size effects become relevant especially as the temperature approaches $T_{\mathrm{c}} \cdot{ }^{24,27,30-34}$ The latter studies report values in the range $\tilde{T}_{\mathrm{c}}=1.294-1.326$ with significant scatter if more data from different groups are considered. ${ }^{29}$ The accurate determination of the critical parameters is not at the heart of the current work, rather we want to discuss the influence of $r_{\text {cut }}$ on capillary criticality below. Fitting of Eq. (7) to the simulation data gives values in the range $\tilde{T}_{\mathrm{c}}=1.29-1.30$ for the simulations including the LRC, depending on which values are included in the fit (orange diamonds in Fig. 1 (B)), in fair agreement with the literature. The orange dashed line shown in Fig. 1 (B) indicates $\tilde{T}_{\mathrm{c}}=1.298$ determined via the critical scaling of the surface tension to be discussed below. We solve Eqs. (5) and (6) numerically resulting in $\tilde{T}_{\mathrm{c}}=1.313$ and $\tilde{\rho}_{\mathrm{c}}=0.310$ in perfect agreement with the values set by Johnson et al. when fitting the MWBR equations, ${ }^{22}$ shown as solid horizontal line in Fig. 1 (B). Simulations of this work using the truncated and shifted potential are shown in Fig. 1 (B) as purple diamonds together with simulation data from Panagiotopoulos (white triangles). ${ }^{23}$ The dash-dotted line in Fig. 1 (B) shows the corresponding mean field correction to the MWBR model for the truncated and shifted LJ potential. Excellent agreement with the simulation data is observed over the full cutoff range.

The critical density can be estimated using the rectilinear diameter

$$
\frac{\rho_{\mathrm{sat}}^{1}+\rho_{\mathrm{sat}}^{\mathrm{v}}}{2}=\rho_{\mathrm{c}}+A\left(T-T_{\mathrm{c}}\right)
$$

where $A$ is another fitting parameter. The rectilinear densities obtained from the GEMC simulations in this work are shown as colored hollow symbols and the predictions of the MWBR equation as dashed lines in Fig. 1 (A). The critical density is independent of $r_{\text {cut }}$ in our simulations within the statistical uncertainty and in line with the MBWR mean field correction for a sufficiently large cutoff, see Supplemental Figure S1. The mean field corrected EOS, however, underestimates $\rho_{\mathrm{c}}$ for $r_{\text {cut }} \lesssim 3.5 \sigma$ (see Supplemental Figure S1), whereas $T_{\mathrm{c}}$ is well predicted also for small $r_{\text {cut }}$ in Fig. 1 (B). In other words, $T_{\mathrm{c}}$, corresponds to the energy scale to bring a particle from the liquid to the vapor phase (or vice versa) and is strongly affected by the missing tail energy, Eq. (2); $\rho_{\mathrm{c}}$ on the other hand is dominated by short range correlations and thus turns out to be nearly independent of $r_{\text {cut }}$. 

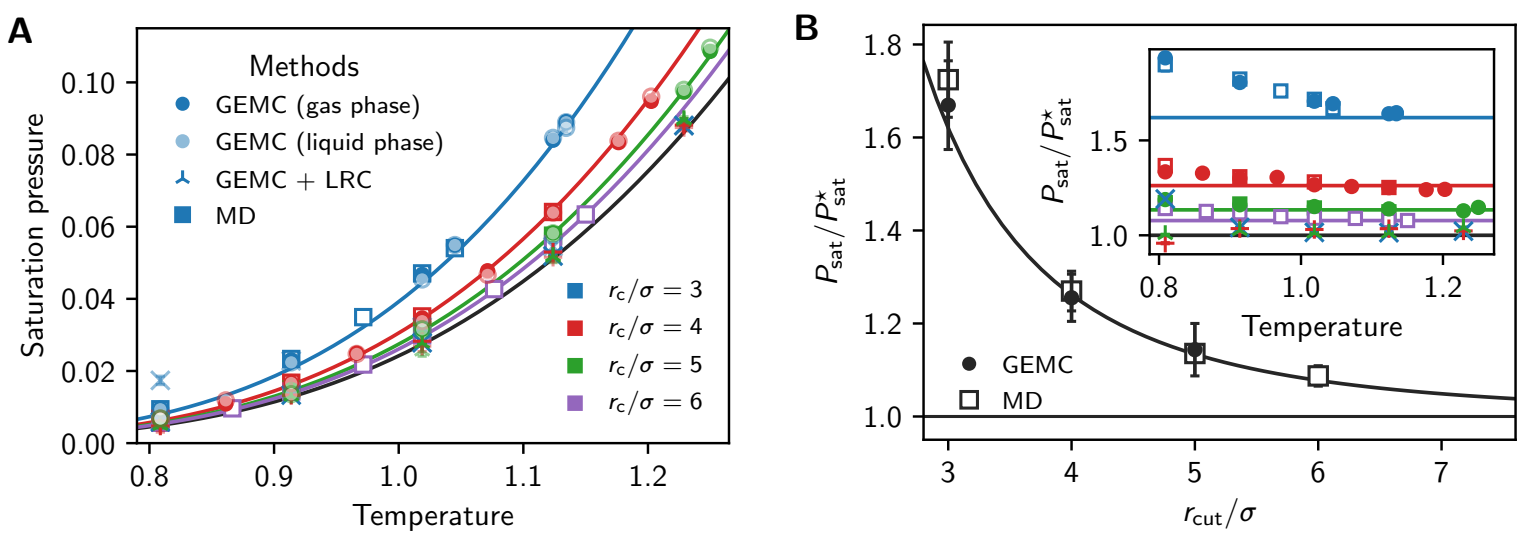

Figure 2. Liquid/vapor saturation pressure. (A) Saturation pressure $\tilde{P}_{\text {sat }}$ as a function of the temperature. The solid black line denotes the Antoine law, Eq. (10), with parameters taken from Kofke. ${ }^{31}$ Solid squares denote results from direct MD simulations of liquid/vapor coexistence for $r_{\text {cut }} / \sigma=3,4,5$ and 6 , respectively (blue to purple symbols). Circles denote results from GEMC simulations, crosses include the long-range correction Eq. (3). (B) Change of the saturation pressure for finite cutoff $r_{\text {cut }}$, normalized to $P_{\text {sat }}^{\star}$ using the parameters obtained by Kofke ${ }^{31}$ for simulations including the long-range correction. Solid line denotes the shift in the saturation pressure due to the missing long-range contribution in the mean field approximation, Eq. (11). The inset shows the same data as in (a) but now also normalized to $P_{\text {sat }}^{\star}$

\section{B. Liquid/vapor saturation pressure}

As seen in the previous section, the choice of the cutoff $r_{\text {cut }}$ has drastic consequences on the thermodynamic properties when using a truncated and shifted LJ potential. Considering pore filling and capillary condensation, the precise knowledge of the liquid/vapor saturation pressure $P_{\text {sat }}$ is of great importance. In this work, we evaluate the pressure from the configurations of the GEMC simulations from the virial microscopic expression via the trace of the pressure tensor ${ }^{1}$

$$
\tilde{P}=\operatorname{Tr}\left[\tilde{T} \tilde{\rho}+\frac{1}{3 \tilde{V}} \sum_{i<j} \tilde{\mathbf{F}}_{i j} \cdot \tilde{\mathbf{r}}_{i j}\right],
$$

which we analyze separately in the vapor and the liquid volumes; equality of both pressures is also a measure for proper equilibration of the simulations. The simulation data are shown in Fig. 2 (A) and reveal a monotonous increase of $\tilde{P}_{\text {sat }}$ with increasing temperature and decreasing cutoff. We additionally perform Molecular Dynamics (MD) simulations of the liquid-vapor interface in the $N V T$ ensemble with $N=3750 \mathrm{LJ}$ particles (shown as squares in Fig. 2 (A)) in a cubic box of length $\tilde{L}=16$. To exclude finite size effects, we checked for selected data points that simulations 
with $N=15000$ and $\tilde{L}=32$ yield identical results. All MD simulations were carried out using LAMMPS $^{35}$ with a time-step $\tilde{t}=t \sqrt{\varepsilon /\left(m \sigma^{2}\right)}=0.9 \cdot 10^{-4}$ and periodic boundary conditions. The data obtained from GEMC and MD simulations agree excellently, thus strengthening that our results are robust w.r.t. finite size effects. Compared to GEMC simulations, MD yields the advantage of straight-forward parallelization and fast phase-space sampling for a LJ fluid. The saturation pressure of the LJ fluid with LRC is well described by an Antoine law,

$$
\log \tilde{P}_{\text {sat }}^{\star}=A^{\prime}-\frac{B^{\prime}}{C^{\prime}+\tilde{T}}
$$

where $A^{\prime}=3.31885 B^{\prime}=7.31828$ and $C^{\prime}=0.039433$ are established fit parameters obtained by Kofke, ${ }^{31}$ c.f. black line in Fig. 2 (A). The simulation data including the LRC (crosses in Fig. 2 (A)) agree well with Eq. (10) with significant deviations only for $r_{\text {cut }}=3 \sigma$ at low temperatures (see inset of Fig. 2 (B)). Colored lines in Fig. 2 (A) and inset of (B) show the corresponding mean field prediction following from Eq. (3),

$$
\tilde{P}_{\text {sat }}=\tilde{P}_{\text {sat }}^{\star}\left(1-\frac{32}{9} \pi\left(\frac{\tilde{\rho}_{\text {sat }}}{\tilde{\rho}_{\text {sat }}^{\star}}\right)^{2}\left[\frac{1}{\tilde{r}_{\text {cut }}^{9}}-\frac{3}{2 \tilde{r}_{\text {cut }}^{3}}\right]\right),
$$

where we have used $\tilde{\rho}_{\text {sat }} / \tilde{\rho}_{\text {sat }}^{\star}=1$ in good agreement with the simulation data shown in Supplemental Figure S1.

The average normalized saturation pressure $P_{\text {sat }} / P_{\text {sat }}^{\star}$ for the different values $r_{\text {cut }}$ is shown in Fig. 2 (B) together with the prediction from Eq. (11). The temperature-dependence shown in the inset of Fig. 2 (B) reveals that deviations appear at low temperatures and with decreasing cutoff, corresponding to the breakdown of the assumption $g(r)=1$ for $r>r_{\text {cut }}$. As discussed in detail in the Supplemental Material, the relative error $\left(\tilde{P}_{\text {sat }}-\tilde{P}_{\text {sat }}^{\star}\right) / \tilde{P}_{\text {sat }}^{\star}$ is typically about $5 \%$ and, thus, by all practical means negligible for the interpretation of adsorption isotherms to be discussed below. Significant deviations of up to $20 \%$ appear, however, for $r_{\text {cut }}=3 \sigma$ at low temperatures, see the blue data in the inset of Fig. 2 (B). Importantly, the overall agreement does not improve if an extended Antoine law for $P_{\text {sat }}(T)$ is used, see Supplemental Figure S2, strengthening the breakdown of the mean field approximation for $r_{\text {cut }} \lesssim 3 \sigma$.

\section{Surface tension}

The liquid-vapor surface tension $\gamma$ further characterizes the thermodynamic properties of the fluid and becomes relevant for the analysis of phase coexistence in confinement to be discussed below. For the Lennard-Jones potential, $\gamma$ has been studied extensively since more than 
four decades and it has soon been realized that tail corrections similar to Eq. (3) contribute significantly. $5,6,26,36-40$ However, most studies report the surface tension explicitly taking into account some tail correction, for which usually a flat interface with a tanh-shaped density profile is assumed and the corresponding long range correction is added a posteriori. Note that this approach explicitly relies on the assumption that the densities $\rho_{l}$ and $\rho_{v}$ in the liquid and vapor phase do not change significantly with $r_{\text {cut }}$, in strong contrast with simulation data and the mean field corrected EOS shown in Fig. 1 (A). In a general approach, the tail energy equivalent to Eq. (2) due to the missing interactions behind a distance $r_{\text {cut }}$ must be taken into account on the fly in simulations, and the explicit terms are available only in simple geometry. ${ }^{7}$ We thus consistently use the truncated and shifted potential Eq. (4) in this work, allowing for interfaces of arbitrary geometry.

From our MD simulations we extract the surface tension from the normal and tangential components of the virial pressure Eq. (9), ${ }^{1}$

$$
\gamma=2\left(P_{\mathrm{N}}-P_{\mathrm{T}}\right)
$$

where the factor 2 accounts for the fact that due to the use of periodic boundary conditions we have two surfaces in our system and $\tilde{\gamma}=\gamma \sigma^{2} / \varepsilon$. Simulation results $\tilde{\gamma}\left(\tilde{T}, r_{\text {cut }}\right)$ are summarized in Fig. 3. Near the critical points one expects the surface tension to vanish as ${ }^{42}$

$$
\gamma(T)=\gamma_{0}\left(1-T / T_{\mathrm{c}}\right)^{2 v} .
$$

A fit to all simulation data and using $T_{\mathrm{c}}\left(r_{\text {cut }}\right)$ as determined in the previous section shown in the inset of Fig. 3 (A) indeed yields the critical exponent of the correlation length $v=0.63$, in perfect agreement with the three-dimensional lattice gas model. ${ }^{43-45}$ The convergence of the simulation data onto Eq. (13), shown as solid lines in Fig. 3 (A), again justifies the use of the mean field approximation in our transformation of the EOS to the truncated-shifted potential with cutoff. We explicitly checked that the obtained surface tension does not depend on the system size by doubling the lateral area to $2 \tilde{L}=36$ for $r_{\text {cut }}=4 \sigma$, shown as red crosses in Fig. 3 (A). We also include in Fig. 3 the data by Mecke et al. ${ }^{38}$ for $r_{\text {cut }}=5 \sigma$ and $\tilde{L} \approx 12$ as green crosses. Their data point at $\tilde{T}=1.15$ is slightly off, suggesting that, when approaching the critical point $\tilde{T}_{\mathrm{c}}\left(r_{\text {cut }}=5 \sigma\right)=1.28$, finite size effects might get important, which is consistent with the deviation of our data for $r_{\text {cut }}=3 \sigma$ at high temperature (blue data in Fig. 3). Contrary, the strong deviation from the scaling behavior Eq. (13) at low temperature for this small cutoff is attributed to a failure of the mean field model as discussed above. 
A

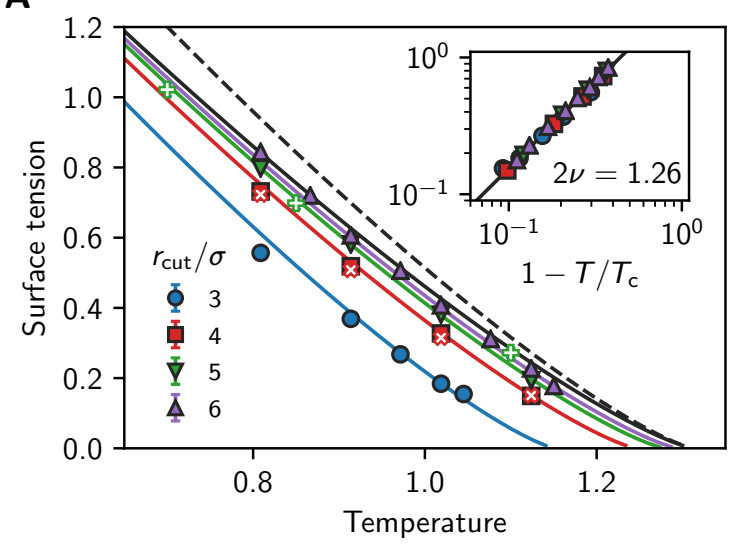

B

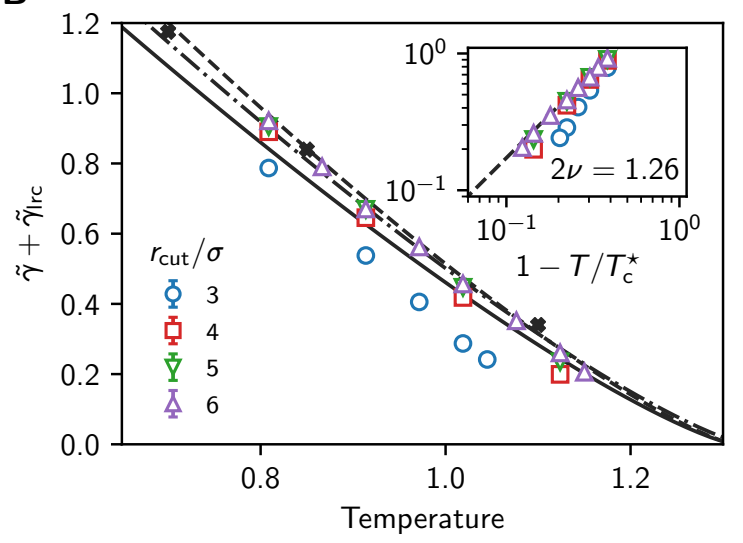

Figure 3. Surface tension of the LJ liquid/vapor interface. (A) MD Simulation results (filled symbols) using the truncated-shifted potential for various cutoff values. Simulations are performed in a box of size $L \times L \times 2 L$ with $L / \sigma=16$. Red crosses show additional simulations for $L / \sigma=32$ and $r_{\text {cut }} / \sigma=4$. Green crosses denote simulations by Mecke et al. for $r_{\text {cut }} / \sigma=4$ in a box $L \times L \times 3 L$ with $L / \sigma \approx 12 .{ }^{38}$ Inset shows the hyperscaling relation Eq. (13) with the critical exponent $v=0.63$, solid line shows the slope $2 v=1.26$ as guide to the eye. Rescaling Eq. (13) with the cutoff-dependent critical temperature the colored solid lines are obtained. Solid Black line extrapolates $T_{\mathrm{c}}=1.313$ to the full LJ potential from the EOS. (B) Simulated surface tension including the long range correction Eq. (14). The critical temperature $T_{\mathrm{c}}^{\star}=1.298$ was fitted using $v=0.63$ (inset), the corresponding curve according to Eq. (20) is shown as dashed black lines in (A) and (B). Dash-dotted lines denote fitting results of Mecke et al. with $v=0.63$ and $T_{\mathrm{c}}=1.325$ for comparison. ${ }^{38}$ Black symbols denote reference data from the National Institute of Standards and Technology using Transition Matrix Monte Carlo including long range LJ interactions via the lattice sum method and with rigorous finite size scaling. ${ }^{41}$

For a classical van der Waals fluid the interfacial density profile on a mean field level has the form $\rho(z)=\rho_{\mathrm{c}}-\Delta \rho \tanh (z /(2 \xi))$, where $\Delta \rho=\rho_{l}-\rho_{v}$ and $\xi$ is the interfacial thickness. Using a tanh profile $\rho(z)$ and the Kirkwood-Buff formula for the surface tension, ${ }^{46}$ Blokhuis et al. have derived the long range contribution of the LJ potential assuming that $\xi$ is independent of $r_{\text {cut }}{ }^{6}$

$$
\tilde{\gamma}_{\mathrm{lrc}}=12 \pi(\Delta \rho)^{2} \int_{0}^{1} \mathrm{~d} s \int_{r_{\mathrm{cut}}}^{\infty} \mathrm{d} r r^{-3}\left(3 s^{3}-s\right) \operatorname{coth}\left(\frac{s r}{2 \xi}\right) .
$$

Values of the interfacial width $\xi$ are obtained from the simulation data and shown in Supplemental Fig. $S 3$ (A). For $r_{\text {cut }} \geq 4 \sigma$ the width $\xi$ moderately depends on $r_{\text {cut }}$ for $\tilde{T}<1$, consistent with the 
tail corrected surface tension $\tilde{\gamma}+\tilde{\gamma}_{\text {Irc }}$ for our simulation data in Fig. 3 (B). Contrary, $r_{\text {cut }}=3 \sigma, \xi$ differs by about $10 \%$ from the larger cutoffs and the corresponding surface tension in Fig. 3 (B) does not converge to the data points for larger cutoff. We fix the critical exponent $v=0.63$ for the tail corrected data with $r_{\text {cut }} \geq 5 \sigma$ to obtain the critical temperature $T_{\mathrm{c}}^{\star}=1.298$ in the inset of Fig. 3 (B), in excellent agreement with the GEMC simulation results using the critical exponent of the order parameter in Fig. 1 (B). Whereas the precise value of $T_{\mathrm{c}}$ for a $\mathrm{LJ}$ fluid is still under debate, the Ising critical exponents have been determined with high precision and we suggest that, using a priori corrections ${ }^{7}$ with sufficient cutoffs, the estimated surface tension is reliable and accurate and can thus actually be used to determine $T_{\mathrm{c}}$ with high precision. Note that we employ the MWBR EOS to determine $T_{\mathrm{c}}\left(r_{\text {cut }}\right)$, in good agreement with the simulation data in Fig. 1 (B) and satisfactory convergence of the simulation data in Fig. 3 (B). Thus the parametrization of the MWBR EOS using $T_{\mathrm{c}}=1.313$ yields reliable estimates $T_{\mathrm{c}}\left(r_{\text {cut }}\right)$, although we here suggest a critical temperature of $T_{\mathrm{c}}^{\star}=1.298$. Using $T_{\mathrm{c}}$ one obtains a slightly higher exponent $v^{\star}=0.65$, see Supplemental Fig. $S 3(B)$, i.e., considering the uncertainty in $T_{\mathrm{c}}$ the analysis of the surface tension scaling does not give reliable estimates for the critical exponent.

\section{Fugacity coefficient of a $\mathbf{L J}$ fluid}

In grand-canonical Monte Carlo (GCMC) simulations, the chemical potential $\mu$ of a fictive ideal gas reservoir is prescribed and particle exchange and translocation moves are performed according to a Metropolis criterion. The reduced chemical potential $\tilde{\mu}=\mu / k_{\mathrm{B}} T$ is linked to the fugacity via

$$
\tilde{\mu}=\ln \tilde{f}+\ln \tilde{\Lambda}^{3},
$$

with the de Broglie length $\Lambda=\tilde{\Lambda} \sigma=\sqrt{h^{2} /\left(2 \pi m k_{\mathrm{B}} T\right)}$ and the fugacity is rescaled according to $\tilde{f}=f \varepsilon / \sigma^{3}$. In general, the pressure $P$ for an interacting system differs from $f$ and one thus defines the fugacity coefficient $\varphi=f / P$, where for $\varphi=1$ the ideal gas law is recovered. Note that the same correction must be applied in a volumetric adsorption measurement, where the adsorbed amount is often calculated from an ideal gas law at measured pressure $P .{ }^{47}$ Typically, the density and pressure are assumed to be sufficiently low such that $\varphi \approx 1$, yet the correction factor for nitrogen adsorption at $77 \mathrm{~K}$ and close to $P_{\text {sat }}$ is about 5\%, in line with the MWBR EOS and our simulation results discussed below. ${ }^{48}$ The use of the fugacity in common adsorption isotherms does not have a major effect as increasing temperature at fixed pressure makes the ideal gas approxi- 

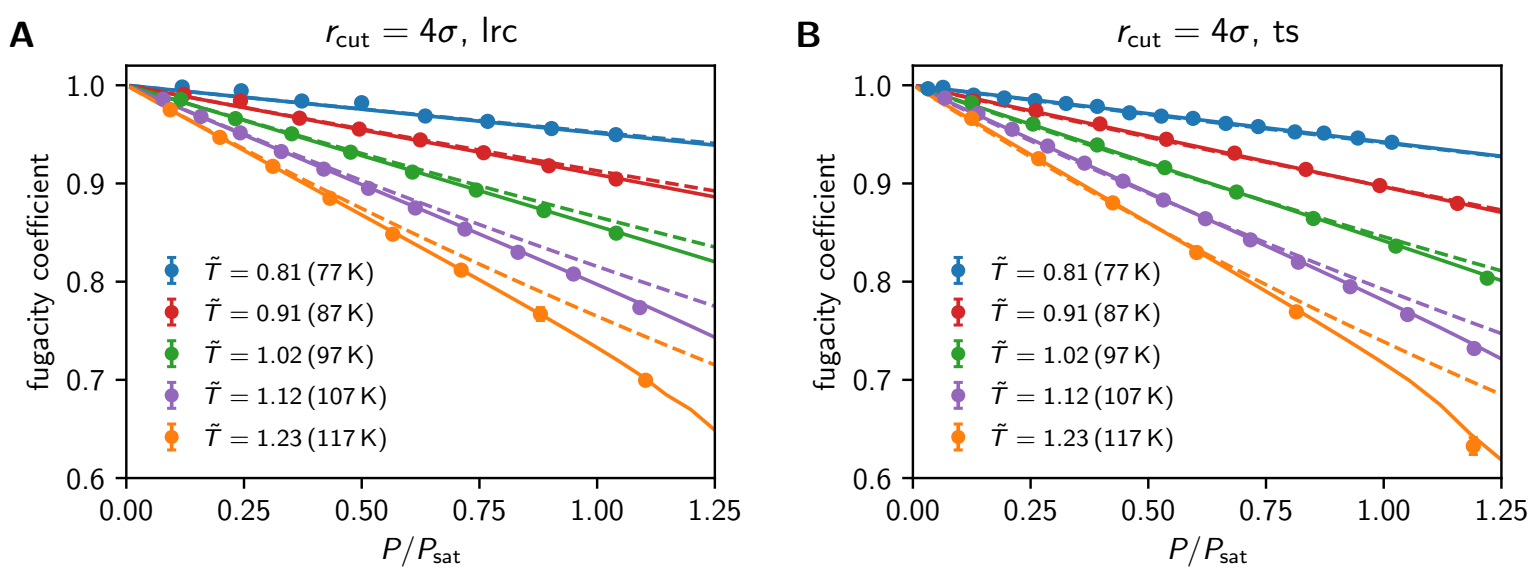

Figure 4. Fugacity coefficient of a LJ liquid. Simulation data for $r_{\text {cut }}=4 \sigma$ using (A) the LJ potential Eq. (1) together with the LRC and (B) the truncated-shifted potential Eq. (4) obtained from GCMC simulations. Solid lines show the fugacity coefficient, determined using Eq. (16) and the MWBR equations. Dashed lines denote the corresponding result from the virial expansion up to second order. Temperature in brackets show physical units for the nitrogen parameters, c.f. Table I.

mation better. However, it is common practice to normalize the isotherms to the corresponding saturation pressure. For increasing temperature, $P_{\text {sat }}$ increases (cf. Fig. 2 (A)) as well as the vapor saturation density $\rho_{\mathrm{v}}$ (cf. Fig. 1 (A)), i.e., $\varphi$ will depart from the ideal gas behavior at $P_{\text {sat }}$ for increasing temperature.

The fugacity coefficient follows from the (inverse) equation of state $\rho(P)$, which we determine by numerical inversion of the MWBR equations, as

$$
\ln \varphi=\int_{0}^{P}\left\{\frac{1}{\rho k_{\mathrm{B}} T}-\frac{1}{P^{\prime}}\right\} \mathrm{d} P^{\prime}=B_{2} P+\mathscr{O}\left(P^{2}\right),
$$

where in the last step we performed a virial expansion up to second order and $B_{2}$ is the second virial coefficient, see the Supplemental Material for details. For a LJ liquid, $B_{2}$ can be evaluated analytically in terms of modified Bessel functions, ${ }^{49}$ and we show in Supplemental Figure S4 that the second order virial EOS indeed describes $\rho(P)$ accurately for the LJ gas at moderate temperatures $\tilde{T} \lesssim 1$. For practical purposes, the virial expansion yields the advantage that $B_{2}$ can be measured straightforwardly both in experiments as well as in molecular simulations (see Supplemental Figure $S 4$ for a comparison). Yet, at temperatures close to $T_{\mathrm{c}}$ the difference amounts to more than $10 \%$, which is clearly not negligible.

Figure 4 shows the fugacity coefficients for various temperatures and a selected cutoff $r_{\text {cut }}=4 \sigma$ 
obtained from GCMC simulations using LAMMPS (A) with the LRC and (B) using the cut and shifted potential, respectively. A cubic simulation box of length $\tilde{L}=26.6$ was chosen, corresponding to up to 8200 particles. We checked that our results are not subject to finite size effects by running selected simulations at box length $2 \tilde{L}$. Solid lines in Fig. 4 are obtained from the MBWR equation of state via Eq. (16), while dashed lines result from the virial expansion up to second order with the analytical virial coefficient. In all cases, the simulation data are perfectly described by the MWBR parameters, whereas with increasing temperature higher order terms in the virial expansion have to be included. One expects the virial expansion to improve with increasing temperature, but we plot in Fig. 4 the fugacity coefficient vs. the vapor/liquid saturation pressure, which according to Eq. (10) increases exponentially with temperature, and thus leads to stronger deviations from the ideal gas behavior with increasing temperature.

\section{ADSORPTION ISOTHERMS}

Having assessed the bulk properties of a LJ liquid, we now study the adsorption of a LJ fluid in an amorphous slit pore. In detail, we consider $\mathrm{N}_{2}$ at $77 \mathrm{~K}$, like is commonly used for the characterization of porous solids. ${ }^{11-17}$ A computational model of amorphous silica was obtained by quenching from cristobalite as described in Ref. ${ }^{50}$, see Fig. 5 (A). A finite slit pore of width $H$ is obtained by expanding the cubic unit cell of length $a=38.5 \AA$ in the $z$-direction such that $L_{z}=a+\left(H+2 \sigma_{\mathrm{N}_{2} / \mathrm{O}}\right)$, where we account for the finite size of nitrogen such that for $H \gtrsim 0$ a $\mathrm{N}_{2}$ molecule fits into the slab, see Fig. 5 (B). The unit cell was replicated three times in the $x$-direction and a reservoir of length $100 \AA$ was added on both sides, see Fig. 5 (C). Periodic boundary conditions in all directions are used and for $r_{\text {cut }}=5 \sigma$ the system also was replicated in $y$-direction in order to exclude interactions with periodic images. Molecular nitrogen is modeled as a single LJ sphere with parameters $\sigma=3.75 \AA$ and $\varepsilon / k_{\mathrm{B}}=95.2 \mathrm{~K} .{ }^{51}$ The Lennard-Jones parameters for the oxygen atoms of the silica substrate are $\sigma_{\mathrm{OO}}=2.7 \AA$ and $\varepsilon_{\mathrm{OO}} / k_{\mathrm{B}} T=230 \mathrm{~K}$, and following previous simulations of nitrogen adsorption in silica pores, we neglect interactions with silicon and hydrogen atoms of the substrate because of the low polarizability. ${ }^{52}$ The cross-parameters for the interaction between the nitrogen molecules and the oxygen atoms of the pores were determined using the Lorentz-Berthelot mixing rules as $\sigma_{\mathrm{N}_{2} / \mathrm{O}}=3.225 \AA$ and $\varepsilon / k_{\mathrm{B}}=147.97 \mathrm{~K}$ (Table I). We have previously shown that this LJ model can be used successfully for capturing the global ex-

perimental behavior upon adsorption and desorption in silica nanopores. ${ }^{53}$ All simulations were 
performed for at least $10^{6} \mathrm{MC}$ steps per nitrogen molecule of which $10 \%$ were discarded as equilibration. To minimize boundary effects of the finite-sized pores, the adsorbed amount $\Gamma$ has been determined only in the central pore region of length $a$, c.f. Fig. 5.

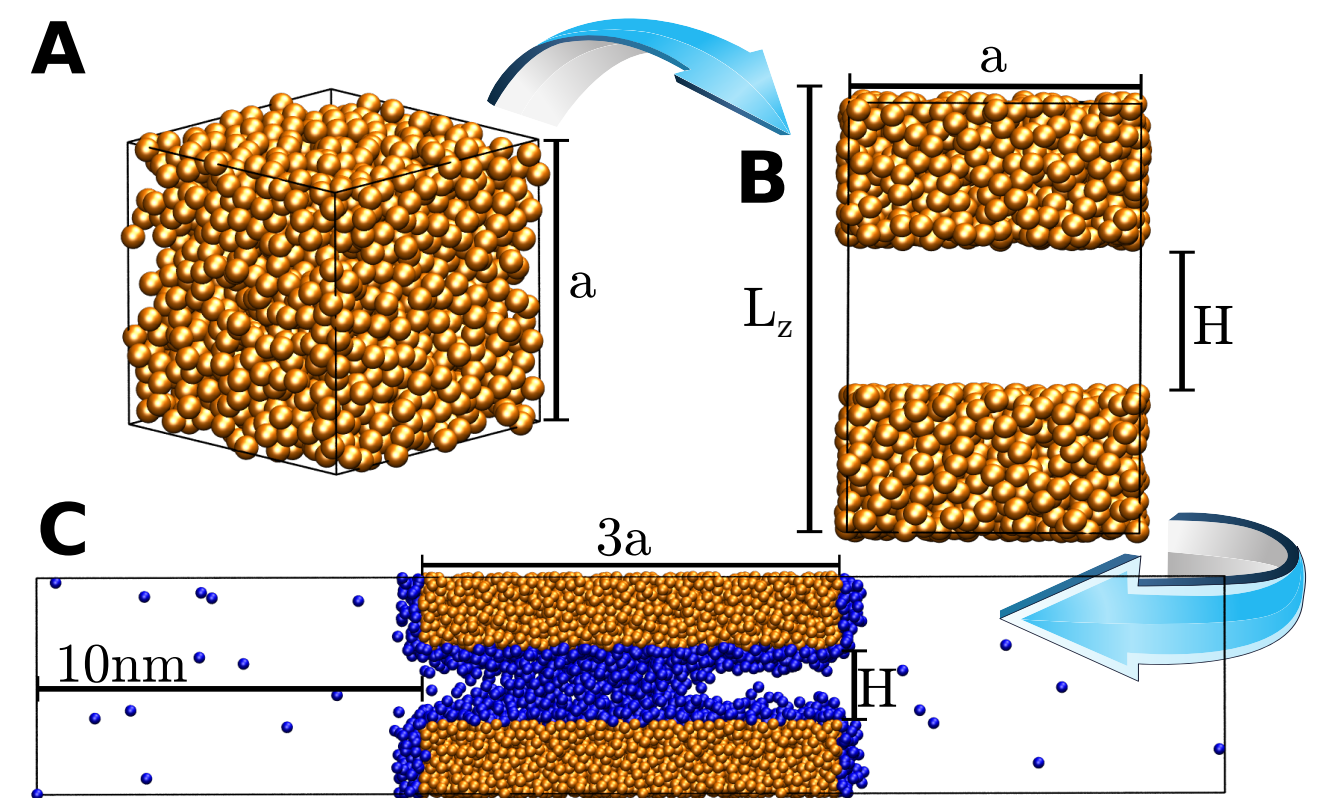

Figure 5. GCMC simulations. (A) Molecular model of amorphous silica as obtained by quenching from cristobalite. ${ }^{50}$ In our simulations, only oxygen atoms (orange spheres) interact with molecular nitrogen, shown as blue spheres in (C). (B) A slit pore of width $H$ is obtained by extending the periodic box in the $z$-direction according to $L_{z}=a+\left(H+2 \sigma_{\mathrm{N}_{2} / \mathrm{O}}\right)$. (C) Typical molecular configuration obtained by means of Grand Canonical Monte Carlo simulations at the onset of capillary condensation for $\mathrm{N}_{2}$ (blue spheres) adsorbed at $T=77 \mathrm{~K}$ and $P=0.7 P_{\text {sat }}$ in a finite silica slit pore of width $H=1.2 \mathrm{~nm}$ with an external reservoir of length $10 \mathrm{~nm}$ on each side.

In Figure 6 we report the adsorption/desorption isotherms for three cutoff values $r_{\text {cut }}=3,4$ and $5 \sigma$ and three pore widths $H=1.2,2.2$ and $3.2 \mathrm{~nm}$. In all figures, the adsorbed amount $\Gamma$ has been normalized to the number of molecules in the central filled pore at $P_{\mathrm{sat}}(T), \Gamma_{0}$, and plotted $v s$. the reduced pressure $P / P_{\text {sat }}(T)$. Considering only the data for $\tilde{T}=0.81$ (blue data in Fig. 6), which corresponds to $T=77 \mathrm{~K}$ in our simulations of nitrogen adsorption, the obtained isotherms conform to the typical behavior observed in experiments of adsorption/condensation in nanopores: the adsorbed amount increases continuously in the multilayer adsorption regime until an irreversible jump occurs because of capillary condensation and evaporation of the fluid within the pore. Once 
Table I. Lennard-Jones interaction parameters. Nitrogen molecules ${ }^{\mathrm{b}}$ and the oxygen atoms of the silica substrate $^{\mathrm{b}}$ are modeled as LJ spheres, while cross terms stem from the Lorentz-Berthelot mixing rule.

$$
\begin{array}{c|c}
\sigma_{\mathrm{N}_{2} / \mathrm{N}_{2}} & 3.75 \AA \\
\varepsilon_{\mathrm{N}_{2} / \mathrm{N}_{2}} & k_{\mathrm{B}} \cdot 95.2 \mathrm{~K} \\
\sigma_{\mathrm{N}_{2} / \mathrm{O}} & 3.225 \AA \\
\varepsilon_{\mathrm{N}_{2} / \mathrm{O}} & k_{\mathrm{B}} \cdot 147.97 \mathrm{~K}
\end{array}
$$

${ }^{a}$ Parameters from Ref. ${ }^{51}$

${ }^{b}$ Parameters from Ref. ${ }^{52}$
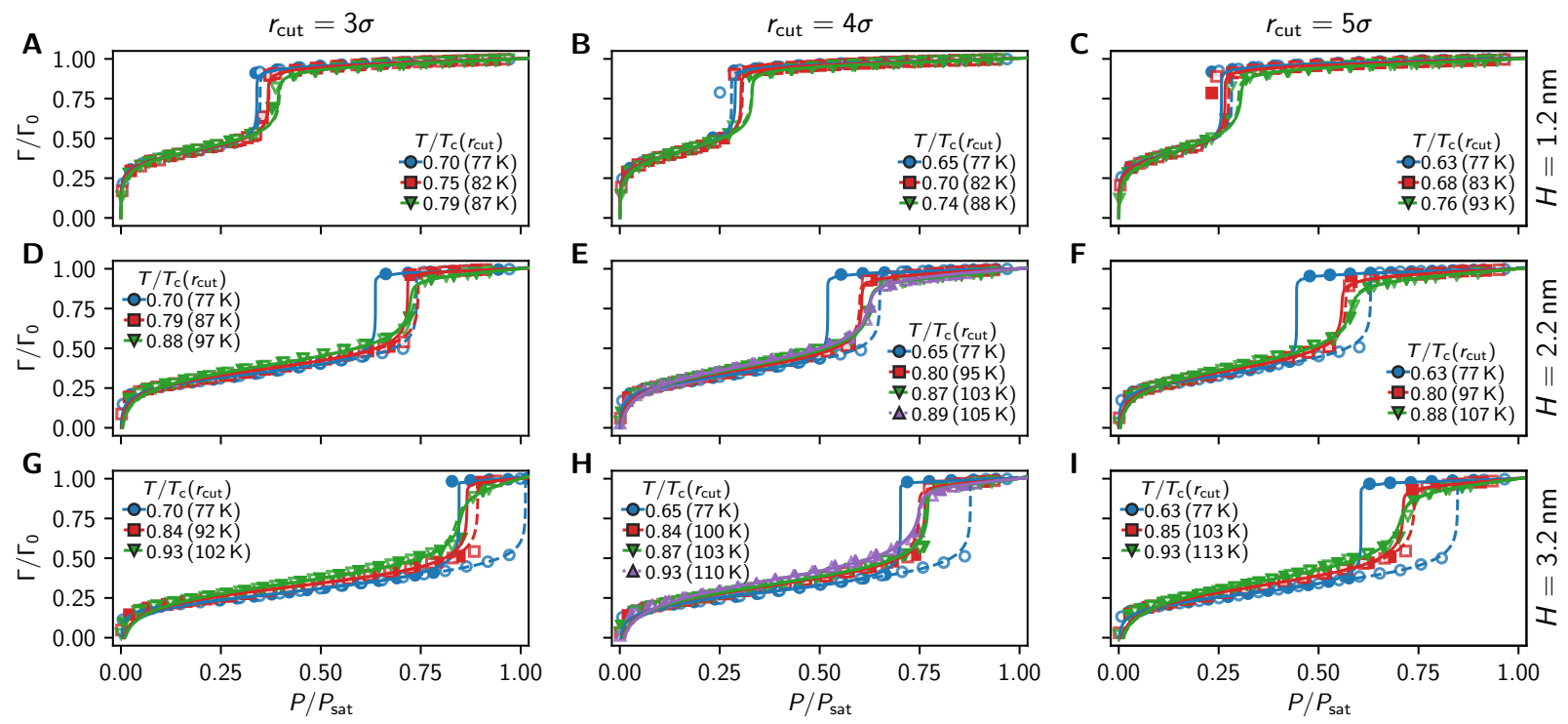

Figure 6. Truncation effect on adsorption isotherms. Adsorption isotherms of nitrogen in silica nanopores are shown as empty symbols, desorption isotherms as filled symbols for $H=1.2 \mathrm{~nm}(\mathrm{~A})-(\mathrm{C})$, 2.2, nm (D)-(F) and 3.2nm (G)-(I). Columns show data for different values $r_{\text {cut }}$. Solid lines denote fits of Eq. (18) to the desorption data, dashed lines to adsorption. Colors denote the temperature normalized to the bulk critical point $T_{\mathrm{c}}\left(r_{\mathrm{cut}}\right)$, corresponding values for the nitrogen parameters are given in brackets for clarity.

the pore is filled, the adsorbed amount changes only slightly upon increasing the pressure due to the small compressibility of liquid nitrogen. With increasing pore width (top to bottom) and increasing cutoff (left to right), the simulation data reveal a pronounced hysteresis loop in Fig. 6. Conceptionally, the presence of condensation and hysteresis can be sketched directly by combining 
the classical Kelvin and Laplace equations, ${ }^{54}$ which shifts the vapor-liquid coexistence pressure according to

$$
P_{\mathrm{v}}(\mu)-P_{1}(\mu)=k_{\mathrm{B}} T \rho \ln \left(\frac{P_{\mathrm{sat}}}{P_{\mathrm{v}}}\right)=\frac{2 \gamma_{\mathrm{lv}}}{H} .
$$

Here, $P_{\mathrm{v}}$ and $P_{1}$ are the pressure in the vapor and the liquid phase, separated in confinement by a curved interface, and $\gamma_{1 v}$ is the liquid-vapor surface tension of the adsorbate. Based on Eq. (17), Derjaguin and Churaev have proposed a thermodynamic model describing both the formation of an adsorbed film at the pore surface and capillary condensation-evaporation. ${ }^{55}$

Considering the simulation data for $77 \mathrm{~K}$ (blue symbols in Fig. 6), the condensation/evaporation pressures decrease inversely with the pore width $H$, as expected from Eq. (17) and the DerjaguinChuraev model ${ }^{56}$ Noteworthy, the width of the hysteresis loop increases with $H$, but also with the cutoff, which we attribute to the increased stability of the adsorbed film for increasing $r_{\text {cut }}$. Whether the adsorption isotherms are determined experimentally or theoretically from molecular simulation, the data points must be fitted with analytical equations for the calculation of thermodynamic properties by numerical integration or differentiation. Instead of relying on expressions based on the underlying physical mechanisms like the Langmuir equation, the BET model or the Derjaguin-Churaev expressions, we employ here a purely phenomenological fit which captures the multilayer adsorption and capillary condensation according to

$$
P(\Gamma)=\frac{w_{1}}{1+\exp \left(-\frac{\Gamma-\Gamma_{1}}{\Delta \Gamma_{1}}\right)}+\frac{w_{2}}{1+\exp \left(-\frac{\Gamma-\Gamma_{2}}{\Delta \Gamma_{2}}\right)} .
$$

Note that Eq. (18) phenomenologically interpolates between a BET isotherm and a saturation at $\Gamma_{1,2} \gg \Gamma$ like in a Langmuir model. Also note that Eq. (18) yields a Langmuir-like linear adsorption regime for $\Gamma \rightarrow 0$, but with an offset $P(\Gamma \rightarrow 0)>0$ that describes the simulation data well for the strongly wetting system considered here. The latter is due to the fact that the silicanitrogen interaction is strong and thus a linear adsorption would be observed only at extremely small pressures.

The fits of Eq. (18) to the simulation data are shown as solid lines in Fig. 6 and reveal a drastic influence of $r_{\text {cut }}$ on the condensation/evaporation pressures as well as on the width of the hysteresis loop. Generally, condensation appears at lower pressures for large cutoff: Increasing $r_{\text {cut }}$ lowers the potential energy according to Eq. (2), and thus increases the relative energy difference between the adsorbed film and the filled pore. Increasing the temperature in all cases results in a transition from a discontinuous pore filling at temperature $T<T_{\mathrm{cc}}$ to a continuous filling at larger 
temperatures $T>T_{c c}$, where $T_{c c}$ is the capillary critical temperature and shall be discussed in the next section. Note that for regular pores such as slits or cylinders, the equilibrium condensation pressure is the one observed on the evaporation branch for wetting pores, whereas condensation occurs in the metastable coexistence region between an adsorbed film and the filled pore due to thermal fluctuations. The condensation pressures increase with the loss in energy due to the cutoff and for our finite number of MC steps condensation on the adsorption branch is not observed for $r_{\text {cut }}=3 \sigma$ at $77 \mathrm{~K}$ for the large pore $H=3.2 \mathrm{~nm}$ in Fig. $6(\mathrm{G})$, as expected from kinetic modeling of the phase coexistence. ${ }^{57}$

\section{CAPILLARY (PSEUDO) CRITICALITY}

A

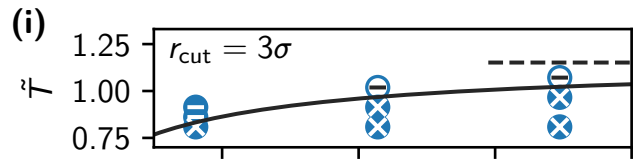

(ii)

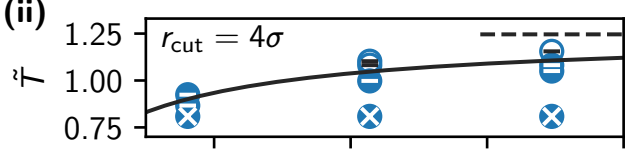

(iii)

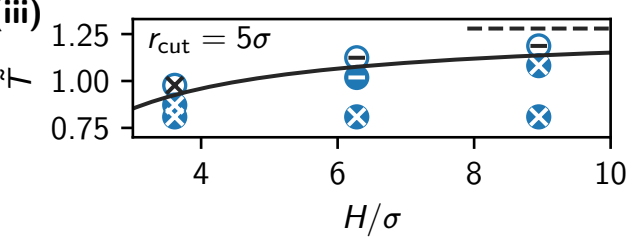

B

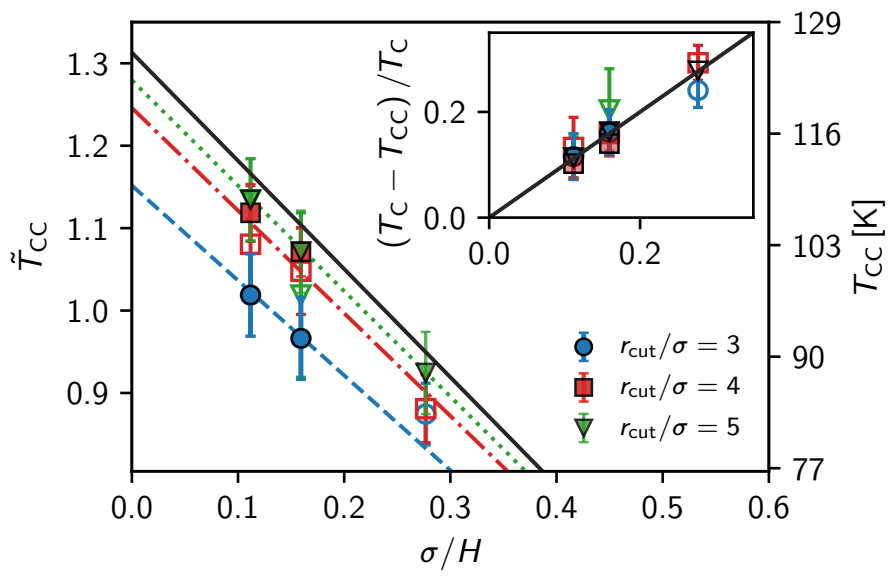

Figure 7. Influence of temperature and cutoff on pore filling. (A) Capillary condensation phase diagram indicating whether pore filling is continuous (open circles) or not (filled circles). Continuity of pore filling has been determined via the FHWM of the compressibility at the condensation pressure for $r_{\text {cut }}=3,4$ and $5 \sigma$ in (i)-(iii), respectively. Symbols indicate if a hysteresis loop is observed (x) or not (-). Dashed lines show the corresponding bulk critical temperature $T_{\mathrm{c}}\left(r_{\mathrm{cut}}\right)$. Solid lines denote the shift of $T_{\mathrm{cc}}$ according to Eq. (20). (B) $T_{\mathrm{cc}}$ as function of the inverse pore width. Solid line denotes the capillary shift of the critical temperature according to Eq. (20) using the LJ potential with long-range correction, broken lines denote the corresponding values $T_{\mathrm{cc}}\left(r_{\mathrm{cut}}\right)$ for the truncated and shifted potential. Filled symbols show $T_{\mathrm{cc}}$, empty symbols the disappearance temperature of the hysteresis loop, $T_{\mathrm{ch}}$. Right scale shows the temperature in physical units for the nitrogen parameters. The inset shows the same data but for the relative change $\Delta T_{\mathrm{c}} / T_{\mathrm{c}}$. 
Capillary condensation (CC) occurs due to cooperative interactions between adsorbed layers, and thus depends on temperature and pore width. Whether capillary condensation in nanopores constitutes a first order transition or not is still debated. ${ }^{58,59}$ Pore filling occurs at a temperature $T_{\mathrm{cc}}$, that is below the bulk critical temperature $T_{\mathrm{c}}$. Above a critical temperate $T_{\mathrm{cc}}(H)<T<T_{\mathrm{c}}$, capillary condensation is suppressed, i.e. pore filling becomes continuous and reversible, which has been confirmed experimentally ${ }^{60,61}$, predicted by density functional theory ${ }^{62}$ and is consistent with our simulation data in Fig. 6. Importantly, $T_{\mathrm{cc}}$ is in general different from the temperature $T_{\mathrm{ch}}$ at which the hysteresis disappears, i.e., capillary condensation still occurs but is reversible as fluctuations are large enough to prevent the system from being trapped in metastable states.

To determine if CC occurs, we evaluate the dimensionless isothermal compressibility

$$
\tilde{\kappa}_{T}=-\frac{\Gamma}{\Gamma_{0}}\left(\frac{\partial\left(\Gamma_{0} / \Gamma\right)}{\partial\left(P / P_{0}\right)}\right)_{T},
$$

from a fit of Eq. (18) to the simulation data (lines in Fig. 4). In a true first-order transition, $\kappa_{T}^{-1}$ vanishes, in contrast to Eq. (18). However, also in experiments, the divergence of the compressibility is usually not observed, which is often attributed to pore-size heterogeneity. ${ }^{61}$ We evaluate the full width at half maximum (FWHM) of the compressibility peak to determine whether CC takes place, i.e., whether the isotherm is discontinuous, or not. The FWHM changes by about an order of magnitude between continuous and discontinuous filling (Supplemental Figure S6) and thus provides a more rigorous definition as the numerical value of $\kappa$, see Supplemental Material for details. The resulting CC phase diagram is shown in Fig. 7 (A) for all pore sizes, temperatures and cutoff values considered. We also indicate by the orange symbols in Fig. 7 (A) the presence of a hysteresis loop if the relative difference between the condensation and evaporation pressures (i.e., the positions of the maxima in $\kappa_{T}$ ) is larger than $2 \%$. As expected, capillary condensation occurs at temperatures lower than the corresponding bulk critical temperature $T_{\mathrm{c}}\left(r_{\text {cut }}\right)$ determined from the MWBR EOS, shown as dashed lines in Fig. 7 (A). For the smallest pore, filling is discontinuous irrespective of $T$, but the hysteresis vanishes with increasing temperature.

The shift $\Delta T_{\mathrm{c}}=T_{\mathrm{c}}-T_{\mathrm{cc}}$ in the critical temperature is from finite size scaling arguments expected to scale as $\Delta T_{\mathrm{c}} / T_{\mathrm{c}} \sim(\xi / H)^{1 / v}$, where $\xi \sim \sigma$ is the correlation length of the density fluctuations and $v$ is a critical exponent. ${ }^{63}$ Using density functional theory (DFT), Evans et al. ${ }^{62}$ derived a relation

$$
\frac{T_{\mathrm{c}}-T_{\mathrm{cc}}}{T_{\mathrm{c}}} \approx \frac{\sigma}{H}
$$

which agrees with the scaling arguments by Nakanishi and Fisher for $v \rightarrow 1 .^{63}$ The DFT result 
given in Eq. (20) is shown in Fig. 7 (A) as solid line with the corresponding values $T_{\mathrm{c}}\left(r_{\text {cut }}\right)$ determined from the MWBR EOS, revealing excellent agreement except for the smallest pore. This disagreement for the small pore is not unexpected as in the DFT calculations the fluid molecules were treated as hard spheres and in such strong confinement correlation effects will become important.

The relevance of the choice of the cutoff becomes clear when the CC data are plotted $v s . \sigma / H$ as proposed by Eq. (20) in Fig. 7 (B). Let us first discuss the drastic shift $\Delta T_{\mathrm{c}}$ due to the confinement if the LJ potential with LRC is employed, black line in Fig. 7 (B). Using the nitrogen simulation parameters (right temperature scale), the critical temperature reduces from its bulk value $T_{\mathrm{c}}=$ $125 \mathrm{~K}$ to $T_{\mathrm{cc}}=102 \mathrm{~K}$ for the pore of width $H=1.2 \mathrm{~nm}$. The simulation data for finite cutoffs using the truncated and shifted potential defined in Eq. (4) are shown as solid symbols, together with corrected bulk temperature $T_{c}\left(r_{\text {cut }}\right)$ obtained from the MWBR EOS (broken lines in Fig. 7 (B)). We also include in Fig. 7 (B) the temperature $T_{\text {ch }}$ at which the capillary hysteresis disappears as empty symbols. The agreement of both $T_{\mathrm{ch}}$ and $T_{\mathrm{cc}}$ with Eq. (20) is remarkable and reveals a shift due to the cutoff ranging from about $3 \mathrm{~K}$ for $r_{\text {cut }}=5 \sigma, 5 \mathrm{~K}$ for $r_{\text {cut }}=4 \sigma$ to a value as large as $15 \mathrm{~K}$ for $r_{\text {cut }}=3 \sigma$. Remarkably, all simulation data converge on the $\Delta T_{\mathrm{c}} \sim H^{-1}$ law, Eq. (20), as we highlight in the inset of Fig. 7 (B).

\section{CONCLUSIONS}

The computational benefit of using a finite cutoff together with the truncated and shifted LJ potential leads to significant changes in the phase diagram, and importantly the critical temperature, even in bulk. Using mean field corrections, we have shown that the capillary critical temperature is well described by the $T_{\mathrm{cc}} \sim T_{\mathrm{c}}(1-\sigma / H)$ law found in DFT calculations and experiments if the consistent value $T_{\mathrm{c}}\left(r_{\text {cut }}\right)$ is used. Care, however, has to been taken in the quantitative comparison of adsorption isotherms: On the one hand, the chemical potential, and thus the fugacity $f$, determines the equilibrium condensation and are straightforwardly accessible both in simulations and volumetric adsorption experiments. On the other hand, it is common practice to present the adsorbed amount $v s$. the pressure $P$ normalized to the vapor/liquid saturation pressure $P_{\text {sat }}$, revealing two possible pitfalls, both in experiments and simulation. First, the saturation pressure depends on temperature, which for a simple fluid like nitrogen is well accounted for by Antoine's equation, and in simulations the pressure correction due to $r_{\text {cut }}$ has to be taken into account. Second, and 
more importantly, $f$ and $P$ are related via the fugacity coefficient $\varphi$. For nitrogen at $77 \mathrm{~K}$ typically $\varphi \approx 1$ is assumed, leading to systematic deviations close to $P_{\text {sat }}$ of about $5 \%$. For higher temperatures as used for the analysis of pore criticality, $\varphi$ becomes non-negligible and is about 0.7 close to $P_{\text {sat }}$. It is therefore of utmost importance to precisely specify if fugacities or pressures are reported.

Summarizing, we have given a comprehensive guide on how to properly take into account truncation effects in molecular simulations whenever the isotropic mean field corrections cannot be applied on the fly, including critical temperature, saturation pressure, surface tension and fugacity coefficient. This allowed us to quantify cutoff effects on modeling adsorption isotherms of fluids in porous media. Performing simulations at different temperatures, we were able to rigorously determine the capillary critical temperature $T_{\mathrm{cc}}$ based on the divergence of the compressibility for a first order transition, which reveals a universal scaling of $T_{\mathrm{cc}}$ with the inverse pore width independent of the detailed interaction parameters.

\section{ACKNOWLEDGMENTS}

We acknowledge funding from the ANR project TAMTAM (ANR-15-CE08-0008-01).

\section{REFERENCES}

${ }^{1}$ M. P. Allen and D. J. Tildesley, Computer Simulation of Liquids (Oxford University Press, 1989).

${ }^{2}$ A. Rahman, Phys. Rev. 136, A405 (1964).

${ }^{3}$ L. Verlet, Phys. Rev. 159, 98 (1967).

${ }^{4}$ D. Levesque, L. Verlet, and J. Kürkijarvi, Phys. Rev. A 7, 1690 (1973).

${ }^{5}$ M. J. P. Nijmeijer, A. F. Bakker, C. Bruin, and J. H. Sikkenk, The Journal of Chemical Physics 89, 3789 (1988).

${ }^{6}$ E. M. Blokhuis, D. Bedeaux, C. D. Holcomb, and J. A. Zollweg, Molecular Physics 85, 665 (1995).

${ }^{7}$ J. Janeček, J. Phys. Chem. B 110, 6264 (2006).

${ }^{8}$ Note that the force $F_{\mathrm{ts}}=-\mathrm{d} \phi_{\mathrm{ts}} / \mathrm{d} r$ used in MD simulations is discontinuous at the cutoff,

$$
F_{\mathrm{ts}}(r)=\left\{\begin{array}{l}
\frac{24 \varepsilon}{r}\left[2\left(\frac{\sigma}{r}\right)^{12}-\left(\frac{\sigma}{r}\right)^{6}\right]-\phi\left(r_{\mathrm{cut}}\right) \delta\left(r-r_{\mathrm{cut}}\right), r \leq r_{\mathrm{cut}} \\
0, \quad r>r_{\mathrm{cut}} .
\end{array}\right.
$$


This discontinuity is usually treated by applying a linear shift in the force over a given range $\Delta r$, however, it turns out that for a reasonable choice of $r_{\text {cut }}$ in most situations the discontinuous force term is of the order of the integration error and therefore can be neglected.

${ }^{9}$ A. Trokhymchuk and J. Alejandre, The Journal of Chemical Physics 111, 8510 (1999).

${ }^{10}$ A. Schlaich, B. Kowalik, M. Kanduč, E. Schneck, and R. R. Netz, in Computational Trends in Solvation and Transport in Liquids, IAS Series, Vol. 28, edited by G. Sutmann, J. Grotendorst, G. Gompper, and D. Marx (Forschungszentrum Jülich GmbH, Jülich, 2015) pp. 155-185.

${ }^{11}$ P. I. Ravikovitch, S. C. O. Domhnaill, A. V. Neimark, F. Schueth, and K. K. Unger, Langmuir 11, 4765 (1995).

${ }^{12}$ K. Morishige, H. Fujii, M. Uga, and D. Kinukawa, Langmuir 13, 3494 (1997).

${ }^{13}$ F. Rouquerol, J. Rouquerol, and K. Sing, in Adsorption by Powders and Porous Solids, edited by F. Rouquerol, J. Rouquerol, and K. Sing (Academic Press, London, 1999) pp. 191-217.

${ }^{14}$ M. Kruk and M. Jaroniec, Chem. Mater. 13, 3169 (2001).

${ }^{15}$ K. Morishige and M. Ito, J. Chem. Phys. 117, 8036 (2002).

${ }^{16}$ M. Thommes, B. Smarsly, M. Groenewolt, P. I. Ravikovitch, and A. V. Neimark, Langmuir 22, 756 (2006).

${ }^{17}$ C. Fan, V. Nguyen, Y. Zeng, P. Phadungbut, T. Horikawa, D. D. Do, and D. Nicholson, Microporous and Mesoporous Materials SI: Characterization of Porous Solids X, 209, 79 (2015).

${ }^{18}$ A. Z. Panagiotopoulos, Molecular Physics 61, 813 (1987).

${ }^{19}$ A. Z. Panagiotopoulos, N. Quirke, M. Stapleton, and D. J. Tildesley, Molecular Physics 63, 527 (1988).

${ }^{20}$ M. G. Martin, Molecular Simulation 39, 1212 (2013).

${ }^{21}$ B. Smit, P. D. Smedt, and D. Frenkel, Molecular Physics 68, 931 (1989).

${ }^{22}$ J. K. Johnson, J. A. Zollweg, and K. E. Gubbins, Molecular Physics 78, 591 (1993).

${ }^{23}$ A. Z. Panagiotopoulos, Int J Thermophys 15, 1057 (1994).

${ }^{24}$ B. Smit, The Journal of Chemical Physics 96, 8639 (1992).

${ }^{25}$ C. D. Holcomb, P. Clancy, S. M. Thompson, and J. A. Zollweg, Fluid Phase Equilibria 75, 185 (1992).

${ }^{26}$ V. G. Baidakov, G. G. Chernykh, and S. P. Protsenko, Chemical Physics Letters 321, 315 (2000).

${ }^{27}$ M. Dinpajooh, P. Bai, D. A. Allan, and J. I. Siepmann, The Journal of Chemical Physics 143, 114113 (2015).

${ }^{28}$ J. J. Nicolas, K. E. Gubbins, W. B. Streett, and D. J. Tildesley, Molecular Physics 37, 1429 
(1979).

${ }^{29}$ M. Thol, G. Rutkai, A. Köster, R. Lustig, R. Span, and J. Vrabec, Journal of Physical and Chemical Reference Data 45, 023101 (2016).

${ }^{30}$ A. Lotfi, J. Vrabec, and J. Fischer, Molecular Physics 76, 1319 (1992).

${ }^{31}$ D. A. Kofke, The Journal of Chemical Physics (1993), 10.1063/1.465023.

${ }^{32}$ J. J. Potoff and A. Z. Panagiotopoulos, The Journal of Chemical Physics 109, 10914 (1998).

${ }^{33}$ J. M. Caillol, The Journal of Chemical Physics 109, 4885 (1998).

${ }^{34}$ M. G. Martin and J. I. Siepmann, J. Phys. Chem. B 102, 2569 (1998).

${ }^{35}$ S. Plimpton, Journal of Computational Physics 117, 1 (1995).

${ }^{36}$ G. A. Chapela, G. Saville, S. M. Thompson, and J. S. Rowlinson, J. Chem. Soc., Faraday Trans. 273,1133 (1977).

${ }^{37}$ C. D. Holcomb, P. Clancy, and J. A. Zollweg, Molecular Physics 78, 437 (1993).

${ }^{38}$ M. Mecke, J. Winkelmann, and J. Fischer, J. Chem. Phys. 107, 9264 (1997).

${ }^{39}$ J. Vrabec, G. K. Kedia, G. Fuchs, and H. Hasse, Molecular Physics 104, 1509 (2006).

${ }^{40}$ H. Watanabe, N. Ito, and C.-K. Hu, The Journal of Chemical Physics 136, 204102 (2012).

${ }^{41}$ V. Shen, D. Siderius, W. Krekelberg, and H. Hatch, eds., NIST Standard Reference Simulation Website, NIST Standard Reference Database No. 173 (National Institute of Standards and Technology, Gaithersburg MD, 20899, 2006).

${ }^{42}$ J. S. Rowlinson and B. Widom, Molecular theory of capillarity (Courier Corporation, 2013).

${ }^{43}$ J. C. Le Guillou and J. Zinn-Justin, Phys. Rev. B 21, 3976 (1980).

${ }^{44}$ J.-H. Chen, M. E. Fisher, and B. G. Nickel, Phys. Rev. Lett. 48, 630 (1982).

${ }^{45}$ A. M. Ferrenberg and D. P. Landau, Phys. Rev. B 44, 5081 (1991).

${ }^{46}$ J. G. Kirkwood and F. P. Buff, J. Chem. Phys. 17, 338 (1949).

${ }^{47}$ S. Brunauer, P. H. Emmett, and E. Teller, J. Am. Chem. Soc. 60, 309 (1938).

${ }^{48}$ W. V. Loebenstein and V. Deitz, J. Research Nat. Bur. Standards 46, 51 (1951).

${ }^{49}$ P. Vargas, E. Muñoz, and L. Rodriguez, Physica A: Statistical Mechanics and its Applications 290, 92 (2001).

${ }^{50}$ F. Villemot, A. Galarneau, and B. Coasne, J. Phys. Chem. C 118, 7423 (2014).

${ }^{51}$ M. W. Maddox, J. P. Olivier, and K. E. Gubbins, Langmuir 13, 1737 (1997).

${ }^{52}$ A. Brodka and T. W. Zerda, The Journal of Chemical Physics 95, 3710 (1991).

${ }^{53}$ B. Coasne, A. Galarneau, F. Di Renzo, and R. J. M. Pellenq, Langmuir 26, 10872 (2010).

${ }^{54}$ E. Charlaix and M. Ciccotti, in Handbook of Nanophysics: Principles and Methods, edited by 
K. D. Sattler (CRC Press, 2010).

${ }^{55}$ B. V. Derjaguin and N. V. Churaev, Journal of Colloid and Interface Science 54, 157 (1976).

${ }^{56}$ B. Coasne, A. Galarneau, R. J. M. Pellenq, and F. D. Renzo, Chemical Society Reviews 42, 4141 (2013).

${ }^{57}$ T. Hiratsuka, H. Tanaka, and M. T. Miyahara, J. Phys. Chem. C 121, 26877 (2017).

${ }^{58}$ R. Valiullin, S. Naumov, P. Galvosas, J. Kärger, H.-J. Woo, F. Porcheron, and P. A. Monson, Nature 443, 965 (2006).

${ }^{59}$ A. O. Parry, C. Rascón, N. B. Wilding, and R. Evans, Phys. Rev. Lett. 98, 226101 (2007).

${ }^{60}$ M. Thommes and G. H. Findenegg, Langmuir 10, 4270 (1994).

${ }^{61}$ K. Morishige and M. Shikimi, J. Chem. Phys. 108, 7821 (1998).

${ }^{62}$ R. Evans, U. M. B. Marconi, and P. Tarazona, The Journal of Chemical Physics 84, 2376 (1986).

${ }^{63}$ H. Nakanishi and M. E. Fisher, J. Chem. Phys. 78, 3279 (1983). 